\title{
A pictorial review of parotid pleomorphic carcinosarcoma radiological findings with clinico-pathologic correlation
}

\author{
A Nada, and C Leiva Salinas \\ Radiology Department, University of Missouri Healthcare, Columbia, MO
}

\section{Purpose:}

Parotid carcinosarcoma is an extremely rare mixed malignant tumor in the head and neck region. Carcinosarcomas have an aggressive characteristic with distant metastasis, commonly to lungs. These tumors are regarded as high-grade tumors. We present a case of parotid pleomorphic carcinosarcoma with review of its characteristic imaging features and correlate these findings with the cytologic and histologic findings including immunohistochemical results.

\section{Case presentation:}

51-year-old male with a history of retinoblastoma during childhood required bilateral eye enucleation followed by external beam radiation treatment. In February 2018, the patient developed left parotid mass. The patient underwent contrast-enhanced CT study which showed left parotid heterogeneously enhancing mass. The mass extends through the stylo-mandibular tunnel, displaces the left parapharyngeal space medially and compresses the left internal jugular vein. Enhanced MRI study was ordered and revealed the large left parotid heterogeneously enhancing mass, infiltrating the left masticator space muscles without mandibular invasion. The mass has been thought to be metastatic deposit. Therefore, PET/CT was ordered to evaluate for other distant metastatic lesions. PET/CT showed avid FDG activity within the mass and multiple ipsilateral cervical lymphadenopathy. This mass was resected and pathologically proven as carcinosarcoma of the parotid gland.

\section{Discussion and conclusion:}

Carcinosarcoma is a rare malignant 'mixed' tumor in the head and neck region. It is defined as a biphasic tumor containing both malignant epithelial and mesenchymal elements. These types of tumors have an aggressive characteristics and are often regarded as a high-grade tumor with distant metastasis occurring in 54\% of patients. They commonly metastasize to the lung. Carcinosarcoma is known to have arisen from a pleomorphic adenoma, which is the most common neoplasm of major salivary glands though in some cases, this type of tumor appears to have originated de novo. Some authors believe that pleomorphic adenomas and carcinosarcomas share a common stem cell, possibly a myoepithelial cell. Malignant transformation of pleomorphic adenoma occurs in around $5-25 \%$ of untreated patients and this encompasses three entities -carcinoma ex pleomorphic adenoma, carcinosarcoma and metastasizing pleomorphic adenoma. The latter two are exceedingly rare.

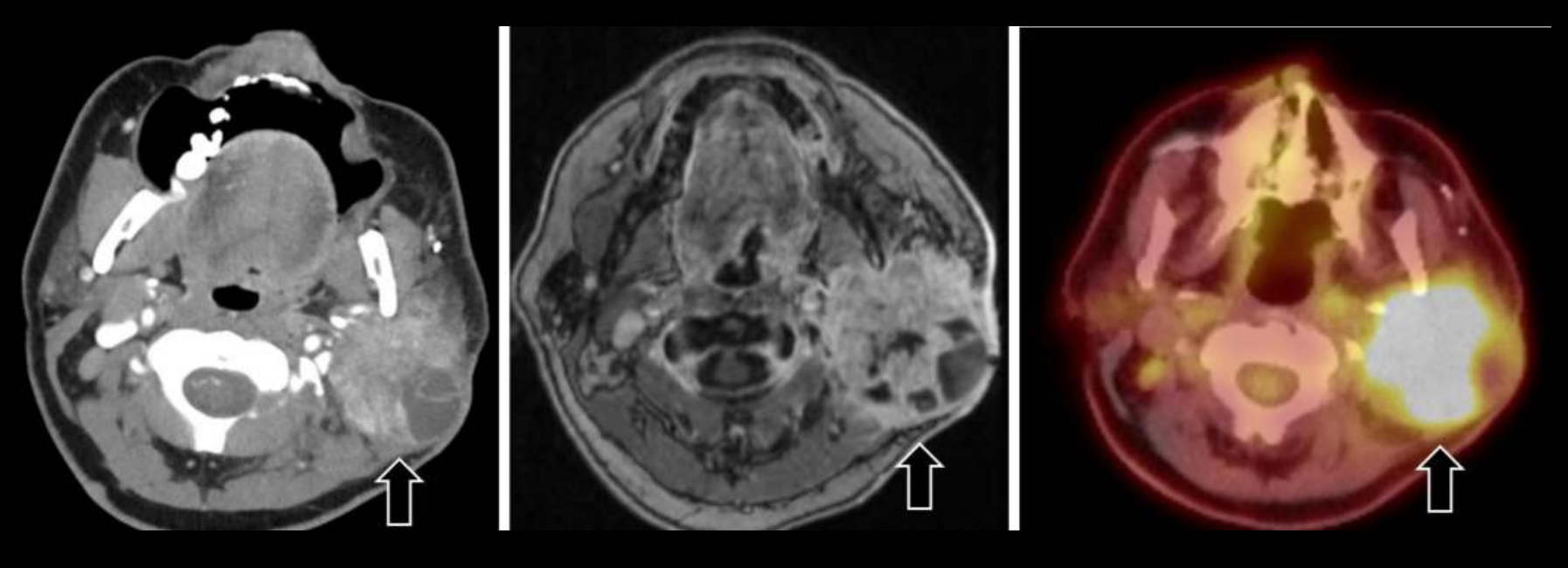

\section{References:}

Keh SM, Tait A, Ahsan F. Primary carcinosarcoma of the parotid gland. Clin Pract. 2011;1(4):e117. Published 2011 Nov 14. doi:10.4081/cp.2011.e117 